\title{
Huella de carbono de una pavimentación con la metodología del ACV y SIMAPRO
}

\section{Carbon footprint of a paving with the ACV and SIMAPRO methodology}

ACEVES-GUTIERREZ, Humberto†*，LÓPEZ-CHÁVEZ，Oscar， MERCADO-IBARRA， Santa Magdalena y AREVALO-RAZO, José Luis

Instituto Tecnológico de Sonora

ID $1^{\mathrm{er}}$ Autor: Humberto, Aceves-Gutierrez / ORC ID: 0000-0001-9916-3114, Researcher ID Thomson: F-8970-2018, CVU CONACYT ID: 2811581

ID $1^{\text {er }}$ Coautor: Oscar, López-Chávez / ORC ID: 0000-0002-0568-2763

ID $2^{\text {do }}$ Coautor: Santa Magdalena, Mercado-Ibarra / ORC ID: 0000-0002-4417-0736 Thomson: H-3386-2018, CVU CONACYT ID: 258533

ID $3^{\text {er }}$ Coautor: José Luis, Arevalo-Razo / ORC ID: 0000-0002-0553-7515, arXiv Author ID: cesarcq, CVU CONACYT ID: 972613

DOI: $10.35429 / J R E .2020 .12 .4 .8 .20$

Recibido 3 de Marzo, 2020; Aceptado Junio 30, 2020

\section{Resumen}

Las actividades que desarrolla el ser humano son las responsables de las emisiones de gases de efecto invernadero que calientan el planeta y generan el cambio climático. El gas más conocido es el $\mathrm{CO}_{2}$, causante del $63 \%$ del calentamiento, el cual está generando consecuencias en los procesos esenciales de nuestra naturaleza, como el caso de los ecosistemas, como los humedales, están en riesgo de desaparecer, el aumento de la temperatura media y la disminución de las precipitaciones, el aumento del nivel del mar, la disminución de los hielos del Ártico. La industria de la construcción es una de las formas mediante la cual el ser humano contamina y una de ellas es debido al crecimiento urbano y la demanda de infraestructura urbano, como las vialidades de los fraccionamientos urbanos. Este estudio tiene como propósito la determinación de los $\mathrm{KG}-\mathrm{CO}_{2} / \mathrm{M} 2$ y los impactos ambientales que se generan en la construcción de un pavimento de carpeta asfáltica en fraccionamiento en Ciudad Obregón Sonora empleando la metodología de Ciclo de Vida (ACV) y el software Simapro 9.0, usando los volúmenes de obra requeridos. Los resultados obtenidos de $13.62080 \mathrm{Kg}-\mathrm{CO}_{2} / \mathrm{M} 2$.

Carpeta asfáltica, Pavimento, GEI, KG-CO $2 / \mathrm{M} 2$

\begin{abstract}
Abtract
The activities carried out by human beings are responsible for the greenhouse gas emissions that heat the planet and generate climate change. The best known gas is $\mathrm{CO}_{2}$, causing $63 \%$ of warming, which is generating consequences in the essential processes of our nature, as in the case of ecosystems, such as wetlands, are at risk of disappearing, the increase in temperature Average and decreasing rainfall, rising sea levels, decreased Arctic ice. The construction industry is one of the ways in which human beings pollute and one of them is due to urban growth and the demand for urban infrastructure, such as urban subdivision roads. The purpose of this study is to determine the $\mathrm{KG}-\mathrm{CO}_{2} /$ $\mathrm{M} 2$ and the environmental impacts that are generated in the construction of an asphalt pavement in urban Development in Ciudad Obregón Sonora using the Life Cycle (ACV) methodology and the Simapro software. 9.0, using the required construction volumes. The results obtained of $13.6280 \mathrm{Kg}-\mathrm{CO}_{2} / \mathrm{M} 2$.
\end{abstract}

Asphalt binder, Pavement, GHG, KG-CO2 / M2

Citación: ACEVES-GUTIERREZ, Humberto, LÓPEZ-CHÁVEZ, Oscar, MERCADO-IBARRA, Santa Magdalena y AREVALORAZO, José Luis. Huella de carbono de una pavimentación con la metodología del ACV y SIMAPRO. Revista de Energías Renovables. 2020. 4-12: 8-20

*Correspondencia al Autor (correo electrónico: haceves_itson@ @otmail.com)

$\dagger$ Investigador contribuyendo como primer autor. 


\section{Introducción}

La construcción es uno de los mayores consumidores de materias primas. El sector cementero es responsable de alrededor del $5 \%$ de las emisiones de $\mathrm{CO}_{2}$, principal gas productor del efecto invernadero y cambio climático, ya que el concreto es el material de construcción más empleado en el mundo y que cada año, la industria del concreto emplea 1.6 billones de toneladas de cemento, y cada una de ellas en su fabricación, emite 1 tonelada de $\mathrm{CO}_{2}$ a la atmósfera. Adicionalmente en el proceso de construcción es común el empleo de maquinaria pesada lo que genera también emisiones de dióxido de carbono, situación que también se presenta con el transporte de los materiales al lugar de la obra lo que constituye un 6-8\% de las emisiones totales de gases de efecto invernadero para una obra (Growingbuildings, 2017).

De la litósfera se extraen el $60 \%$ de los materiales que se emplean en la construcción, también el $50 \%$ de las emisiones de $\mathrm{CO}_{2}$ a la atmósfera provienen de la construcción y uso de los edificios, el $40 \%$ de la energía primaria consumida en el planeta y el $75 \%$ de la electricidad se utiliza en edificaciones, el $20 \%$ del agua dulce es consumida en el uso de los edificios y el $60 \%$ de los residuos sólidos se producen en la construcción y destrucción de los edificios (Casanovas, 2009).

El sector de la construcción comercial y residencial representa el 39\% del dióxido de carbono $\left(\mathrm{CO}_{2}\right)$ emitido a la atmósfera la vez que genera el $30 \%$ de los residuos sólidos y el $20 \%$ de la contaminación de las aguas. Por lo que podríamos concluir que la mitad del $\mathrm{CO}_{2}$ expulsado a la atmósfera está relacionado con la construcción de edificios a lo largo de todas sus fases: construcción, uso y posterior demolición. En consecuencia, el sector de la construcción tiene que desempeñar un papel importante en la reducción de la amenaza del cambio climático. (Growingbuildings, 2017).

Para reducir el impacto ambiental del sector de la construcción es importante el uso de materiales que para fabricarlo no requieran la utilización de combustibles fósiles y se eviten las altas emisiones de carbono como por ejemplo la madera.
Entre los principales gases de efecto invernadero el que más preocupa es el dióxido de carbono por su responsabilidad en el cambio climático y de acuerdo con OECC (2013), la concentración de $\mathrm{CO}_{2}$ (dióxido de carbono) en la atmósfera ha aumentado por la actividad humana, fundamentalmente por el uso de combustibles fósiles y la deforestación, con una menor contribución de la producción de cemento, también señala que las concentraciones actuales de $\mathrm{CO}_{2}, \mathrm{CH} 4$ (metano) y $\mathrm{N}_{2} \mathrm{O}$ (óxido nitroso) exceden sustancialmente el rango de concentraciones registradas en los testigos de hielo durante los últimos 800.000 años y el ritmo de incremento de las concentraciones en la atmósfera de $\mathrm{CO}_{2}, \mathrm{CH} 4$ y $\mathrm{N}_{2} \mathrm{O}$ en el pasado siglo $\mathrm{XX}$ no tiene precedente en los últimos 22.000 años, ya que estas han aumentado desde 1750 , excediendo los niveles preindustriales en $40 \%$, $150 \%$ y $20 \%$, respectivamente(figura 1) y finalmente el $\mathrm{pH}$ del agua oceánica ha decrecido 0,1 desde el comienzo de la era industrial, que corresponde a un aumento del $26 \%$ de concentración de iones hidrógeno.

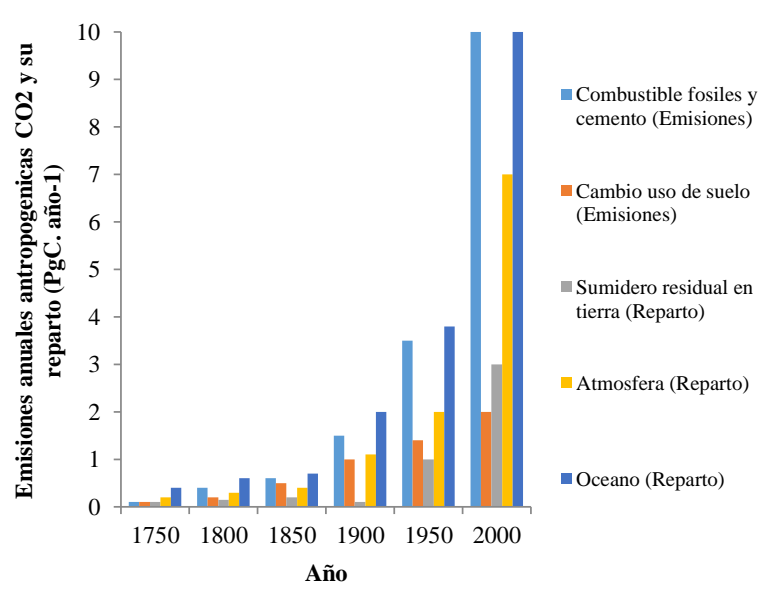

Figura 1 Emisiones anuales antropogénicas $\mathrm{CO} 2$ y su reparto (PgC.año)

Fuente: OECC 2013

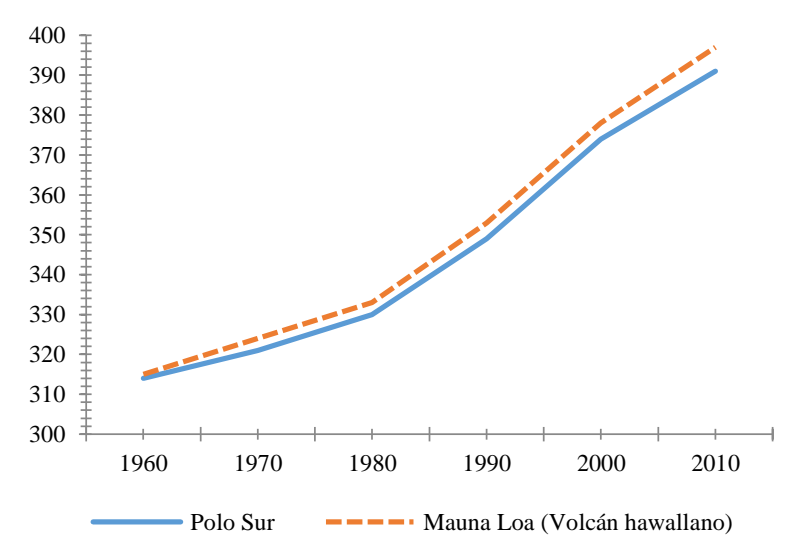

Figura 2 Evolución reciente de la concentración de $\mathrm{CO}_{2}$ (ppm) en la atmósfera

Fuente: AEMA 2013 
De acuerdo a la Agencia Europea del Medio Ambiente (AEMA), las posibilidades de evitar esta interferencia aumentan significativamente si nos situamos por debajo de 450 ppm de CO2eq (Figura 2), las emisiones se han disparado desde mediados del siglo pasado, cuando había unas $310 \mathrm{ppm}$. Por tanto, el momento de actuar es ya, puesto que cada año que pasa sin hacerlo el problema se agrava y las soluciones se complican (y encarecen).

De acuerdo con la figura 3 , las emisiones antropógenas totales de GEI han seguido aumentando entre 1970 y 2010 con mayores incrementos absolutos, entre 2000 y 2010, a pesar del creciente número de políticas de mitigación del cambio climático

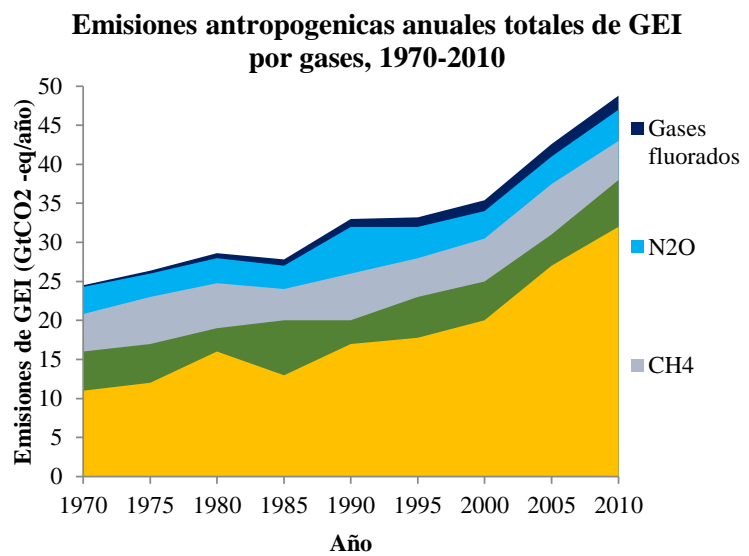

Figura 3 Emisiones antropógenas anuales totales de GEI por gases, 1970-2010

Fuente: IPCC 2015

Las emisiones de $\mathrm{CO}_{2}$ procedente de la combustión de combustibles fósiles y los procesos industriales contribuyeron en torno al $78 \%$ del aumento total de emisiones de GEI de 1970 a 2010, con una contribución porcentual similar para el aumento experimentado durante el período de 2000 a 2010, provocado por el crecimiento económico y el demográfico continuaron siendo los motores más importantes de los aumentos en las emisiones de $\mathrm{CO}_{2}$ derivadas de la quema de combustibles fósiles. (IPCC, 2015)

El impacto ambiental que las industrias tienen sobre el medio ambiente y los recursos naturales ha sido considerable, no tan sólo como resultado del crecimiento de la producción sino también gracias a que dicho crecimiento se concentró en sectores de alto impacto ambiental. (SEMARNAT, 2010).
La industria de la construcción incluye varias fuentes de contaminación que se pueden enmarcar en los distintos aspectos e impactos ambientales propios del sector económico y que modifican el componente abiótico de los ecosistemas, es decir, el suelo, el aire y el agua, Aparicio (2020), como los que se describen a continuación:

El suelo presenta alteración debido a los residuos que provienen que el vertido de desechos y escombros de la construcción con numerosos efectos negativos en el medio ambiente, entre otros: contaminación, utilización excesiva de materiales con la consecuente pérdida de recursos naturales, degradación de la calidad del paisaje y alteración de drenajes naturales, esto genera los impactos de acidificación, la eutrofización y eco toxicidad lo que provoca modificación generada al ecosistema (Acosta, 2002). Adicionalmente, el movimiento de tierra genera alteración de la geomorfología, la pérdida de cobertura vegetal ocasiona procesos de erosión más rápidos y en ocasiones, cuando se usan explosivos para excavaciones en la industria de la construcción, se pueden generar inestabilidad de los taludes (Arenas 2010).

En el aire ocurren alteraciones asociadas al polvo, el ruido, las emisiones de $\mathrm{CO}_{2}$ derivadas del uso de combustibles fósiles, de minerales, trabajos de excavaciones, corte de taludes y operación de máquinas y herramientas, Medineckien, Kazimieras, Turskis (2010) resaltan que el uso de minerales como material de construcción genera finas partículas de polvo durante su proceso de degradación, de acuerdo con la dispersión, los más peligrosos de ellos son partículas duras de la clase $5^{\circ}$, las cuales no son detenidas por las vías respiratorias superiores de los humanos generando problemas en la membrana mucosa de la nariz, la tráquea, los bronquios, que despiertan reacciones inflamatorias y con el tiempo alteraciones crónicas y luego las personas contraen enfermedades de las vías respiratorias, como bronquitis, traqueítis y neumonía (esclerosis difusa de los pulmones). 
Los combustibles fósiles (carbón, gas y petróleo) quemados para producir energía generan las emisiones que provocan cambios climáticos, ya que se libera el dióxido de carbono a las capas más bajas de la atmósfera donde se forma una barrera que atrapa el calor liberado por la tierra, generando lo que se conoce como efecto invernadero. Entre más dióxido de carbono hay en la atmósfera, más calor se acumula y este calentamiento provoca el cambio climático.

En relación con las consecuencias de los diferentes impactos ambientales en el aire algunos estudios han mostrado un importante número de casos de los efectos negativos del ozono, principalmente relacionados con el sistema respiratorio, como problemas en función pulmonar, crecimiento en el número de personas asmáticas, incremento de visitas a urgencias y los ingresos hospitalarios por problemas respiratorios (Ballester, 2005), esto debido a que la concentración de ozono en la atmósfera ha aumentado en casi ocho veces a la concentración atmosférica natural, generando dolor de cabeza, ardor en ojos, infecciones en la piel de las personas (Montaño \& Sandoval, 2007).

La contaminación atmosférica se considera como cancerígena para las personas y se relaciona con el cáncer de pulmón, ahora un nuevo estudio epidemiológico publicado en Environmental Health Perspectives y desarrollado Turner, Krewski, Diver, Pope, Burnett, Jerret, Marshall, Gapstur (2017) quienes realizaron el seguimiento durante 22 años (de 1982 a 2004) a más de 600.000 personas adultas de Estados Unidos, que formaron parte del Estudio II de Prevención contra el Cáncer. El equipo científico relacionó la mortalidad por 29 tipos de cáncer con la exposición residencial a tres contaminantes ambientales: PM2,5, dióxido de nitrógeno (NO2) y ozono (O3).

Las partículas PM2,5 se asociaron con la mortalidad por cáncer de riñón y vejiga, con un aumento del $14 \%$ y del $13 \%$, respectivamente, por cada incremento de exposición de $4.4 \mu \mathrm{g} / \mathrm{m} 3$. Por otro lado, la exposición a NO2 se asoció con la mortalidad por cáncer colorrectal, con un aumento del $6 \%$ por cada incremento de $6.5 \mathrm{ppb}$.
Según datos del Atlas Global del Carbono (2020), un proyecto de la red internacional de científicos Future Earth y la ONU, se obtuvo que en México se registraron 477 megatoneladas de dióxido de carbono $\left(\mathrm{MtCO}_{2}\right)$, por lo que es el país de América Latina que más emitió en 2018, según los datos más recientes, por delante de Brasil con $457 \mathrm{MtCO}_{2} \mathrm{y}$ 196 MtCO2 de Argentina

De acuerdo con la Organización Mundial de la Salud (OMS, 2012), existen pruebas contundentes de que las actividades humanas han trasformado el clima mundial, provocando grandes y graves repercusiones en la salud pública, las cuales están relacionadas con los fenómenos meteorológicos catastróficos, la variabilidad de los climas, que afecta a los suministros de agua y alimentos, los cambios de la distribución de los brotes de enfermedades infecciosas o las enfermedades emergentes relacionadas con los cambios de los ecosistemas. Según la OMS ha estimado un número de muertes atribuible al cambio climático de 160,000 por año y considera que para el año 2050, las olas de calor serán más frecuentes y extremas en algunas zonas de Europa y América del Norte (González, Fernández \& Gutiérrez, 2013).

Esta es la causa por la cual se considera muy importante buscar formas de reducir los gases efecto invernadero (GEI) en especial el $\mathrm{CO}_{2}$ que es el más abundante después del vapor de agua.

Shen, Lu, Yao y Wu (2005) sostienen que la construcción es la principal fuente de contaminación ambiental en comparación con otras industrias, por otro lado, Bravo (2011) señala que el sector de la construcción genera el $36 \%$ de las emisiones de $\mathrm{CO}_{2}$, en la comunidad económica europea y que es la industria que más energía que consume, más residuos genera y utilizando el $60 \%$ de los materiales que se extraen en el continente (Bravo, 2011).

Se ha informado que muy pocos desarrolladores privados y contratistas hacen esfuerzos por considerar al medioambiente $\mathrm{y}$ tomar en cuenta el reciclaje de los materiales de construcción y dan más importancia al tiempo de terminación de la obra. (Poon, Yu, Ng, 2001). 
Zolfagharian, Nourbakhsh, Izarry Ressang,Gheisari (2012) consideran que se debe de reforzar el nivel de conocimiento y la conciencia de todos aquellos que participan en los proyectos de obra, en los impactos ambientales negativos causados por los procesos de construcción. Gangolells, Casals, Gassó,Forcada, Roca,Fuertes (2009), sostienen que al identificar los principales impactos medioambientales de los procesos de construcción se mejora la efectividad de los Sistemas de Gestión Ambiental en las organizaciones que lo tienen, sin embargo, los países desarrollados poseen pocos datos científicos sobre los impactos en el medioambiente producidos por los materiales de construcción y las tecnologías y es difícil tomar opciones informadas que conduzcan hacia la reducción de tales impactos (Pittet y Kotak, 2009).

Es por eso por lo que se debe tomar especial atención al sector de la construcción, para poder prever cuantas emisiones de $\mathrm{CO}_{2}$ se podrían llegar a emitir.

Un fraccionamiento es la división de un predio en manzanas, lotes y/o súper manzanas que requiere del trazo y construcción de una o más vías públicas, así como la ejecución de obras de urbanización que le permitan la dotación de infraestructura, equipamiento y servicios urbanos, debiendo tener todos los lotes acceso a la vía pública (Secretaría de Infraestructura y Desarrollo Urbano, 2018).

En el estado de Sonora en 1990, el 79\% de la población del estado vivía en localidades de 2500 y más habitantes. En 2015, ese porcentaje fue de $87.4 \%$, para una población de 2 millones 932 mil 821 habitantes (Ver Figura 4), según datos publicados por el INEGI (2016).

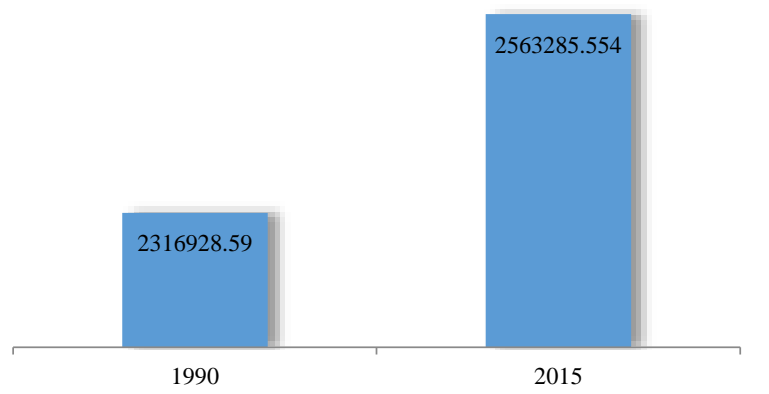

Figura 4 Crecimiento de la población urbana en Sonora Fuente: INEGI 2016
El estado de Sonora se ha caracterizado por la concentración demográfica, producto de los flujos migratorios que han optado por buscar alternativas de mejores empleos e ingresos. Primero las actividades secundarias y ahora las terciarias han sido los principales atractivos de dichos movimientos demográficos, que se han alojado en ciertos puntos del territorio conocidos como centros de población.

Las ventajas de ubicación, así como la densidad y especialidad de las actividades no primarias han provocado diferenciados fenómenos de urbanización en el territorio. Este proceso de urbanización se ha caracterizado también por un intenso uso del suelo medido a través de la densidad de población (SIDUR, 2016).

La población que se concentra en localidades de más de 2,500 habitantes pasó de 451,003 habitantes en 1960, a 2’451,284 habitantes en 2015 quintuplicándose la población en estos 55 años. En términos relativos, la proporción de población estatal que se aloja en localidades mayores a 2,500 habitantes (grado de urbanización), se ha incrementado de $57 \%$ que se registró en 1960 a $87 \%$ en el año 2015, marcando una clara y creciente tendencia de urbanización.

De hecho, más de la mitad de la población de la entidad reside actualmente en ciudades mayores (figura 5) de 100,000 habitantes, con una proporción del $62 \%$ y cerca del $70 \%$ en localidades con poblaciones superiores a los 50,000 habitantes (Programa Sectorial de Infraestructura y Desarrollo Urbano Sustentable, 2016).

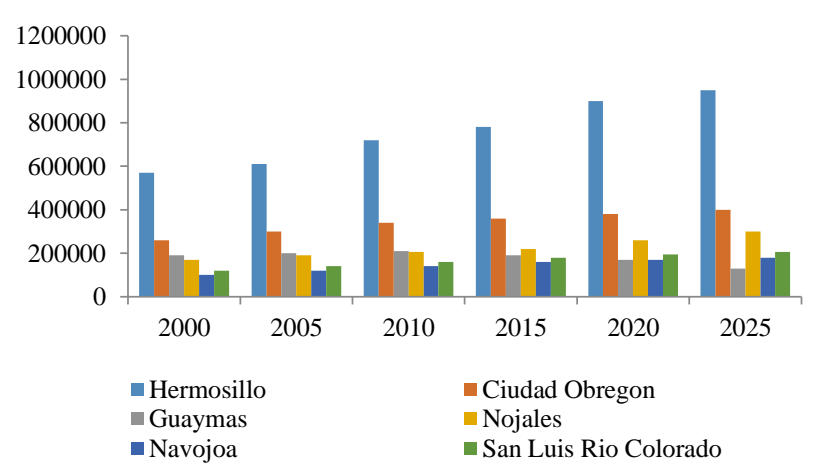

Figura 5 Crecimiento de la población en ciudades mayores a 100000 habitantes

Fuente: Programa Sectorial de Infraestructura $y$ Desarrollo Urbano Sustentables, 2016 
Se entienden por infraestructura urbana las obras que dan el soporte funcional para otorgar bienes y servicios óptimos para el funcionamiento y satisfacción de la comunidad, son las redes básicas de conducción y distribución, como agua potable, alcantarillado sanitario, agua tratada, saneamiento, agua pluvial, energía eléctrica, gas y oleoductos, telecomunicaciones, así como la eliminación de basura y desechos urbanos sólidos (Plan de Desarrollo Urbano de la Ciudad de Chihuahua; Visión 2040, 2009).

Según el Reglamento de Edificaciones del municipio de Cajeme (2015), los desarrollos inmobiliarios, deberán tener las siguientes obras, vialidades, red de agua potable, red de alcantarillado sanitario, sistema para la conducción de aguas pluviales, entre otras obras. El pavimento en vialidades o calles se ha venido desarrollando conforme las necesidades y los descubrimientos de los materiales para construirlos ,comenzando con la aparición de la piedra que utilizó el hombre debido a sus características, siendo Egipto, Grecia e Inglaterra las primeras en desarrollar métodos de pavimentación (Rama, 2006) y en 1905 fue cuando el concreto hidráulico empezó a utilizarse como material de pavimentación en los llamados pavimentos rígidos con el enfoque principal de crear caminos y rutas transitables para el transporte masivo entre las grandes ciudades del país (Patiño, Reyes, Camacho, 2015).

El Pavimento flexible (UNAM, 2010) es una capa de carpeta asfáltica proporcional a la superficie de rodamiento, donde las cargas de los vehículos van hacia las capas inferiores y se distribuyen por medio de fricción y cohesión de las partículas de los materiales; la carpeta asfáltica se pliega a pequeñas deformaciones de las capas inferiores sin que su estructura se rompa. Las capas que forman un pavimento flexible son (Figura 5): carpeta asfáltica, base y sub-base, las cuales se construyen sobre la capa sub-rasante.

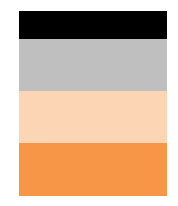

Sub-Rasante

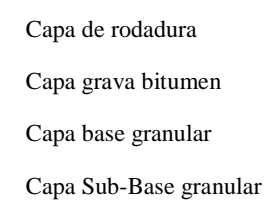

Estructura de pavimento flexible
Figura 6 Estructura de pavimento flexible Fuente: UNAM 2009
En la investigación llevada a cabo se estudiaron trabajos previos relacionados con huella de carbono o las emisiones de $\mathrm{CO}_{2}$ en la Industria de la Construcción donde destacan los siguientes: Evaluación del impacto ambiental de los pavimentos urbanos exteriores. Francalacci (2010), señala que los pavimentos pétreos de concretoin situ, son los que más ocasionan impacto ambiental (213 $\mathrm{kgCO}_{2} / \mathrm{m} 2$, los empedrado con materiales pétreos los que menos impacto generan $14 \mathrm{kgCO}_{2} \mathrm{eq} / \mathrm{m} 2$ ). Mercader, Ramírez, Olivares (2013) abordan un Modelo de cuantificación de las emisiones de $\mathrm{CO}_{2}$ producidas en edificación derivadas de los recursos materiales consumidos en su ejecución, mientras que Güereca Carius Padilla ,Herrera y Paz (2016) presentan un estudio relativo a la Evaluación de la Huella de Carbono con enfoque de Análisis de Ciclo de Vida para 12 Sistemas Constructivos de muros en los cuales encontraron que Muro de concreto de $10 \mathrm{~cm}$ espesor produce $139.67 \quad \mathrm{Kg}-\mathrm{CO}_{2} \mathrm{eq} / \mathrm{m} 2$, mientras que el muro de block hueco o 15x20x40 $63.11 \mathrm{Kg}-\mathrm{Co} 2 \mathrm{eq} / \mathrm{m} 2$, y finalmente Hoyos (2018) en su estudio de determinación de la huella de carbono en la construcción de 3 tipos de Muro, aplicada a casas de interés social, México-Puebla.

De la misma manera se encontró información documentada respecto a las metodologías existentes para la determinación del cálculo de la huella de carbono o emisiones de $\mathrm{CO}_{2}$, algunas de ellas fueron, Metodologías de cálculo de la huella de Carbono y sus potenciales implicaciones para América Latina (CEPAL, 2010) una más denominada 7 metodologías para el cálculo de emisiones de gases efecto invernadero (IHobe,2013) y finalmente Enfoques Metodológicos para el cálculo de la huella de Carbono (Observación de la Sostenibilidad en España, 2017). Con el propósito de aumentar el conocimiento respecto a las emisiones en $\mathrm{KgCO}_{2} \mathrm{eq}$ de la industria de la construcción y tomando en cuenta que los procesos de construcción de las vialidades de un fraccionamiento, con uso de pavimentos flexibles de carpeta asfáltica, son parte de la industria de la construcción específicamente en lo relativo a infraestructura urbana se decidió investigar la cantidad de emisiones que se tienen en la construcción de un pavimento de carpeta asfáltica del fraccionamiento Puente Real de Ciudad Obregón Sonora, de $5 \mathrm{cms}$ de espesor con la estructura de la figura 6 y un área de $128,049.60 \mathrm{~m} 2$ mediante la metodología de ACV y el Software Simapro 9.0.

ACEVES-GUTIERREZ, Humberto, LÓPEZ-CHÁVEZ, Oscar MERCADO-IBARRA, Santa Magdalena y AREVALO-RAZO, José Luis. Huella de carbono de una pavimentación con la metodología del ACV y SIMAPRO. Revista de Energías Renovables. 2020 
Estructura del pavimento

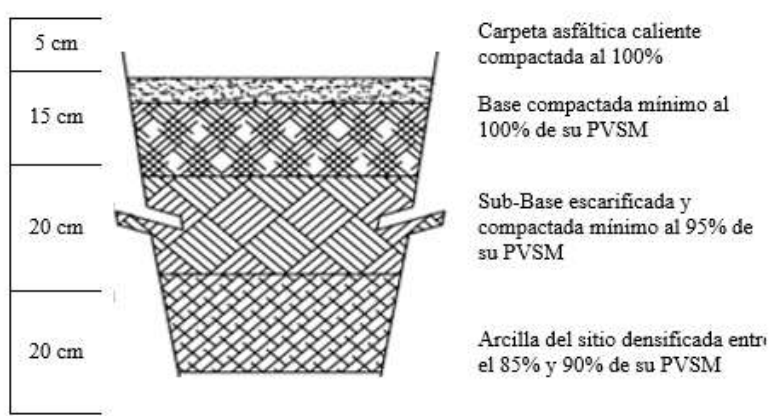

Figura 7 Estructura del pavimento Fuente: Elaboración Propia

El ACV es una herramienta para una mejor comprensión de las dimensiones del perfil ambiental de los productos, procesos y servicios y es adecuada para comparar los impactos medioambientales potenciales de dos o más productos semejantes y de ser necesario puede ser combinado con consideraciones económicas y sociales, y dependiendo de los procesos que contemple el ACV, puede tener distintos alcances, según Badilla, Elizondo, Fernández, Mora, Méndez y Quesada (2015).

- "De la cuna a la tumba": Incluye la extracción de materias primas y el procesado de los materiales necesarios para la manufactura de componente, el uso del producto y finalmente su reciclaje y/o la gestión final. El transporte, almacenaje, distribución y otras actividades intermedias entre las fases del ciclo de vida también se incluyen cuando tienen la relevancia suficiente

- "De la cuna a la puerta": El alcance del sistema se limita desde que se obtienen las materias primas hasta que el producto se pone en el mercado, a la salida de la planta de fabricación

- "De la puerta a la puerta": Sólo se tienen en cuenta los procesos de fabricación

Se consideró como el objeto del estudio la unidad funcional $1 \mathrm{~m} 2$ de pavimento de carpeta asfáltica de $5 \mathrm{~cm} 2$ de espesor con una estructura de la sub-rasante de $20 \mathrm{cms}$, una subbase de $20 \mathrm{cms}$ y una base de $15 \mathrm{cms}$, planteando la Hipótesis de que las emisiones en $\mathrm{KG}-\mathrm{CO}_{2}$ /M2 que producen los pavimentos de carpeta asfáltica se encuentran dentro del rango de 10-25 $\mathrm{KG}-\mathrm{CO}_{2}$.
Para desarrollar el estudio se utilizó la metodología de ACV hasta la fase de construcción (de la cuna a la puerta) eliminado las etapas de uso y de disposición final, a continuación, se muestra la metodología y el Software Simapro 9.0

\section{Metodología}

Para llevar a cabo este proyecto se contó con la participación de un alumno y de profesores investigadores de Ingeniería Civil del Instituto Tecnológico de Sonora, realizando los cálculos pertinentes para la obtención de resultados, los materiales y equipos usados fueron, la hoja electrónica Excel para la visualización de la explosión de insumos y para plasmar los datos creados para cuantificar las emisiones del $\mathrm{CO}_{2}$ generado por la construcción, interpretación de resultados. Las fuentes de información fueron trabajos anteriores relacionados con el proyecto tales como tesis, artículos, revistas científicas, base de datos del gobierno mexicano, bibliografía electrónica en general.

Procedimiento:

1. La selección del fraccionamiento dentro de la ciudad ubicado al norte de Ciudad Obregón Sonora, El plano y los datos de la estructura del pavimento con todos los datos del proyecto anchos y longitudes de las calles (anexo 1).

2. Se procedió al cálculo de las cantidades de obra en cada etapa del proceso constructivo.

3. Se seleccionó la maquinaria de construcción adecuada para cada tipo de trabajo y se determinaron los rendimientos horarios para cada uno de ellos y se obtuvieron las potencias en HP de cada equipo

4. Con cantidades de obra y los rendimientos horarios se determinaron las horas de trabajo necesarias para cada etapa del proceso.

5. Con el número de horas y con la potencia de cada equipo se logró determinar la cantidad de consumo de combustible en litros, en este caso Diésel, el cual se convirtió de energía calorífica en Megajoules MJ

6. Se establecieron como unidad funcional un $\mathrm{m} 2$ de pavimento de $5 \mathrm{~ms}$ (figura 7 ), en el cual se obtuvieron las cantidades de obra requeridas de unidades de volumen, usando los pesos específicos se convirtieron a unidades masa. 
7. Con Google Maps se determinaron las distancias de los lugares de procedencia, obtención de los materiales, como agua, base, sub-base, cemento asfaltico entre otros.

8. Se estableció el esquema sistema producto(figura8)

9. Teniendo toda la información anterior se procedió al uso de Software SIMAPRO de la siguiente manera.

10. Se da de alta el proyecto y se seleccionaron las bibliotecas a usar y se describió el proyecto y se procedió a la integración de los procesos sus entradas y salidas referidas a materiales, procesos empleados y emisiones generadas y se integraron los materiales, los consumos generados por los transportes de los materiales a partir de su lugar de origen, se definieron los procesos del producto y luego en las fases del producto se procedió al ensamblaje, concluido esto se seleccionó la forma de analizar o calcular los impactos, teniendo las opciones de red, árbol, análisis , de las cuales en este proyecto se trabajó solo con las dos últimas del mismo, se seleccionó el producto y la metodología, en este caso ReCiPe 2016 Mid point, World 2010 y a partir de aquí se dieron los resultados en el apartado de caracterización de los impactos.

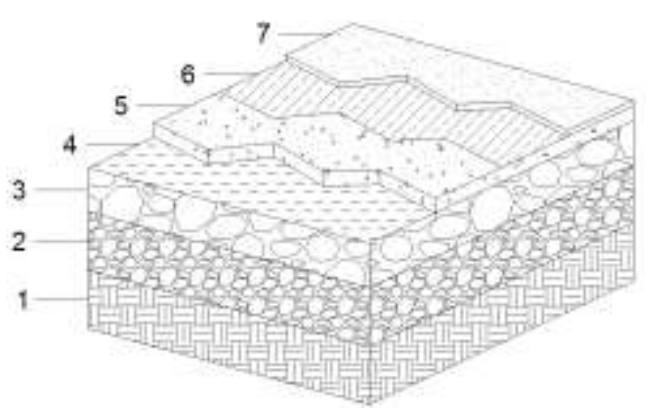

\begin{tabular}{|l|l|c|l|l|}
\hline \multirow{5}{*}{$\begin{array}{l}\text { Materiales } \\
\text { por capas }\end{array}$} & $\begin{array}{l}\text { Capa estabilizadora } \\
\text { granular }\end{array}$ & 1 & Sub-Rasante & $20 \mathrm{~cm}$ \\
\cline { 2 - 5 } & Capa de Impregnación & 4 & Sub-Base & $20 \mathrm{~cm}$ \\
\cline { 2 - 5 } & $\begin{array}{l}\text { Capa de pavimento } \\
\text { asfáltico }\end{array}$ & 5 & Emulse & $15 \mathrm{~cm}$ \\
\cline { 2 - 5 } & Capa de Liga & 6 & Emulsión & $\begin{array}{l}1.15 \\
\mathrm{~L} / \mathrm{m} 2\end{array}$ \\
\cline { 2 - 5 } & Riego de sello & 7 & $\begin{array}{l}\text { Tratamiento } \\
\text { superficial }\end{array}$ & $5 \mathrm{~cm}$ \\
\hline
\end{tabular}

Figura 8 Especificaciones de la estructura de un Pavimento asfáltico de $5 \mathrm{~cm}$ Fuente: Elaboración Propia

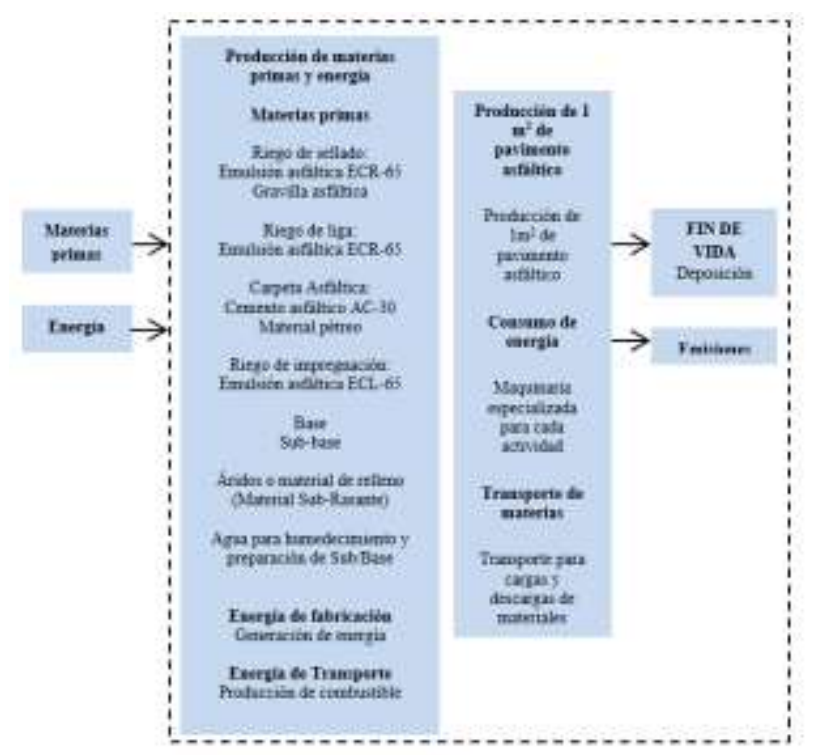

Figura 9 Esquema del sistema -producto Fuente: Elaboración Propia

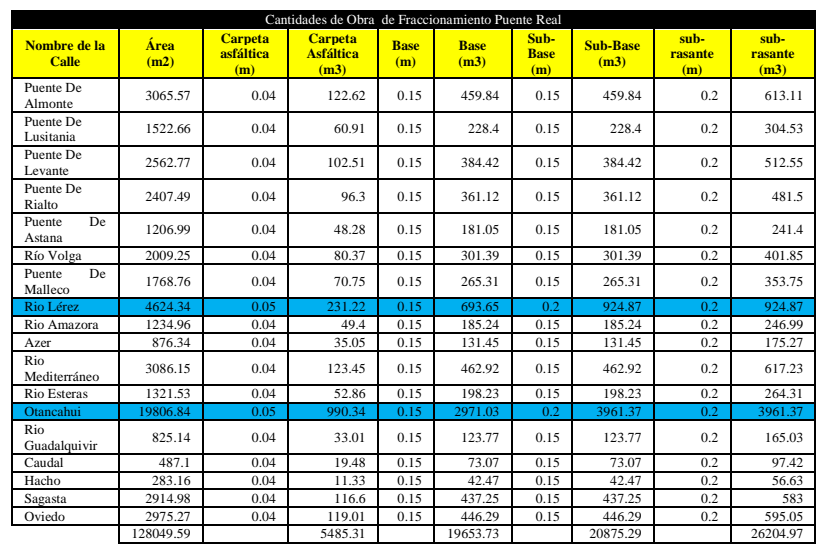

Tabla 1 Cantidades de obra fraccionamiento. Puente Real Fuente: Elaboración Propia

En la tabla 1 se observan los diferentes volúmenes de obra que se determinaron y que fueron necesarios para el estudio destacan el área pavimentada de $128049.99 \mathrm{~m} 2$ y los volúmenes de 26,204.97 m3 de sub-rasante y el volumen de sub-base de 20,875.29

En la tabla 2 se observa que las cantidades de diésel requeridas en cada una de las etapas del proceso constructivo para la pavimentación del Fraccionamiento Puente Real para la sub- base compactada se requiere 0.523 lts/m3, mientras que para la carpeta asfáltica son necesarios $0.0721 \mathrm{lts} / \mathrm{m} 3$. 


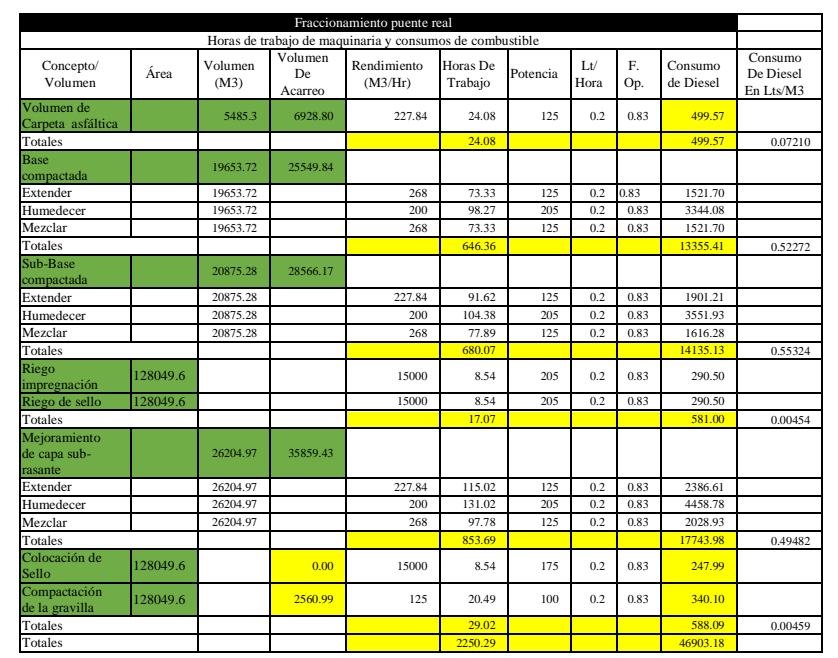

Tabla 2 Horas máquina y consumo combustible Fuente: Elaboración Propia

\begin{tabular}{|l|r|}
\hline \multicolumn{2}{|c|}{$\begin{array}{c}\text { Fraccionamiento puente real poder calorífico en } \\
\text { MJ/M3/fase }\end{array}$} \\
\hline \multicolumn{1}{|c|}{ Fase } & MJ \\
\hline Carpeta asfáltica & 2.59 \\
\hline Base Compactada & 18.79 \\
\hline Volumen de Sub-Base & 19.89 \\
\hline Riego impregnación con petrolizadora & 0.16 \\
\hline Mejoramiento de capa sub-rasante & 17.79 \\
\hline $\begin{array}{l}\text { Compactación de la gravilla Con compactador } \\
\text { liso }\end{array}$ & 0.17 \\
\hline
\end{tabular}

Tabla 3 Poder calorífico Fuente: Elaboración Propia

En el fraccionamiento Puente Real las cantidades de poder calorífico para la unidad funcional de un $\mathrm{m} 2$ destacando el Volumen de Sub-Base con un valor de $19.89 \mathrm{MJ}$ y la base compactada de $18.79 \mathrm{MJ}$, el valor menor es en los riegos de impregnación con .16 MJ.

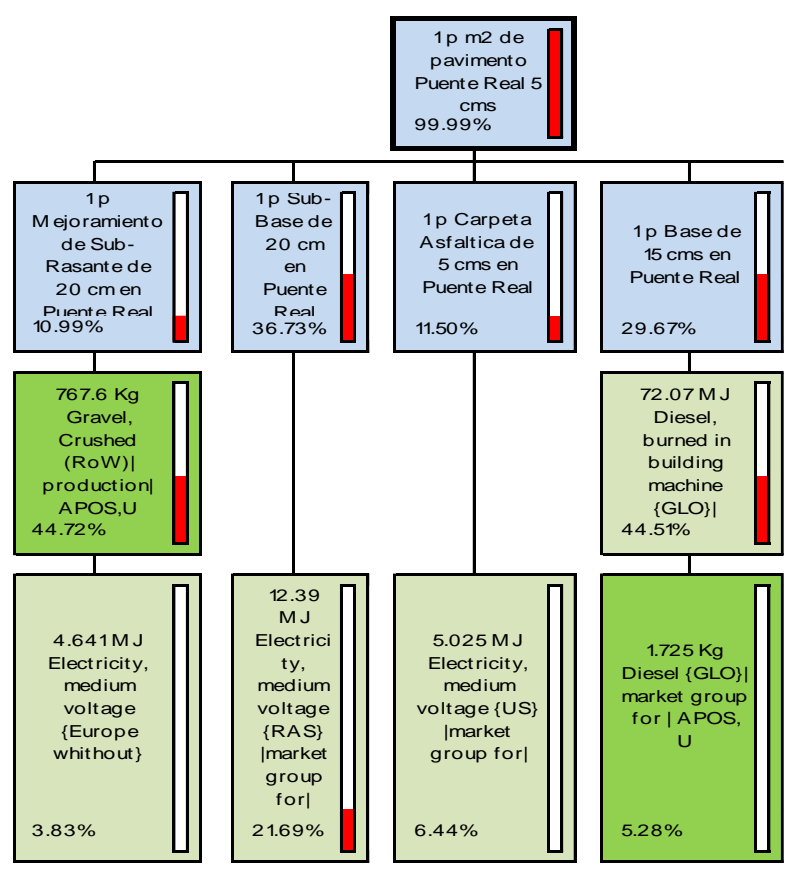

Figura 10 Aportaciones de las etapas del proceso constructivo y de materiales más representativos que intervienen Puente Real $5 \mathrm{cms}$. Simapro 9.0

Fuente: Elaboración Propia

ISSN 2523-2881

ECORFAN ${ }^{\circledR}$ Todos los derechos reservados
La figura 10 muestra en qué porcentajes contribuyen a los impactos ambientales en la pavimentación del fraccionamiento San Rafael, las diferentes etapas del proceso constructivo como el caso de la Subbase con un $36.73 \%$ y $29.67 \%$ de la base y en una subetapa el consumo energético de electricity médium voltaje con $21.69 \%$.

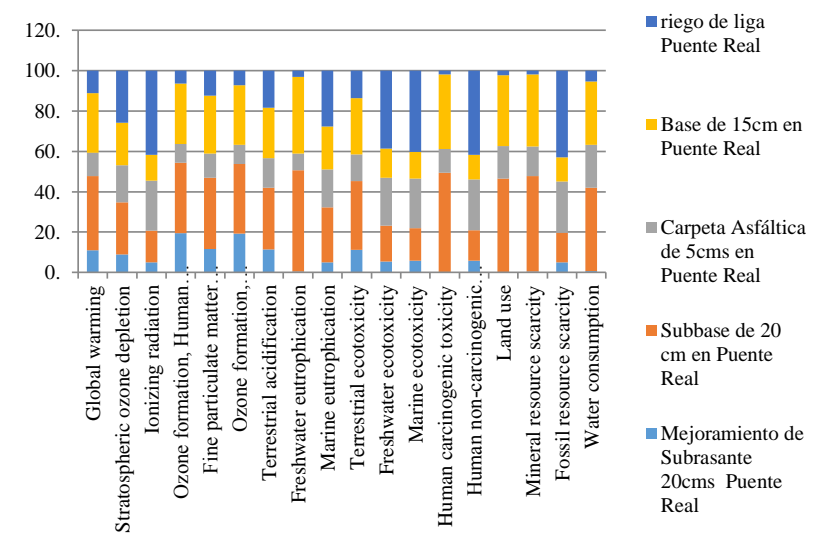

Figura 11 Gráfica de las categorías de los impactos ambientales en las diferentes etapas del proceso constructivo en el fraccionamiento Puente Real, $5 \mathrm{cms}$ de espesor. Simapro 9.0 Fuente: Elaboración Propia

En la figura 11 en la categoría de global warning muestra un comportamiento similar al de Ozone formation y fine particulate matter formation mismas en las que más contribuyen es en sub-base y base, otra categoría que resalta es la Freshwater ecotoxcity y marine ecotoxcity la etapa que más contribuye es la de riego de liga, esto último se ha visto que tiene el mismo resultado que en los demás fraccionamientos.

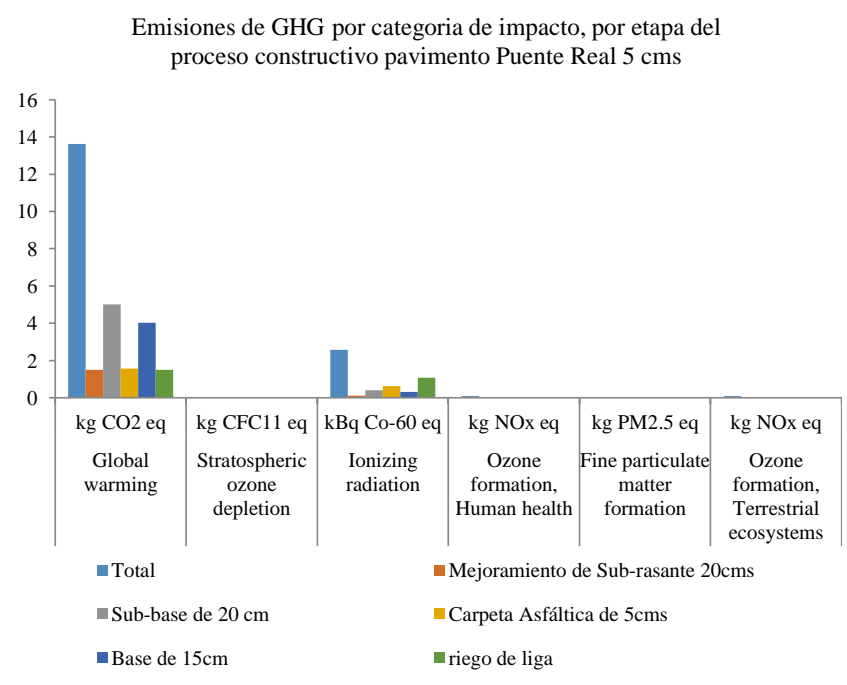

Figura 12 Gráfica de las categorías de los impactos ambientales asociados a los gases efecto invernadero del pavimento de $5 \mathrm{~cm}$ del fraccionamiento Puente Real Simapro 9.0

Fuente: Elaboración propia

ACEVES-GUTIERREZ, Humberto, LÓPEZ-CHÁVEZ, Oscar, MERCADO-IBARRA, Santa Magdalena y AREVALO-RAZO, José Luis. Huella de carbono de una pavimentación con la metodología del ACV y SIMAPRO. Revista de Energías Renovables. 2020 
En la figura 12 se resalta que las emisiones de $\mathrm{CO}_{2}$ relacionadas con Global warming son las más representativas y son del orden de $13.5 \mathrm{Kg}$ de $\mathrm{CO}_{2}$, donde las etapas que más contribuyen son la base y la subbase, otra de las categorías destacable ionizing radiation en $\mathrm{KBqCO}-60 \mathrm{eq}$ que es por el orden total de $1 \mathrm{Kg}$, donde se ve que quién más contribuye es la etapa de Riego de liga.

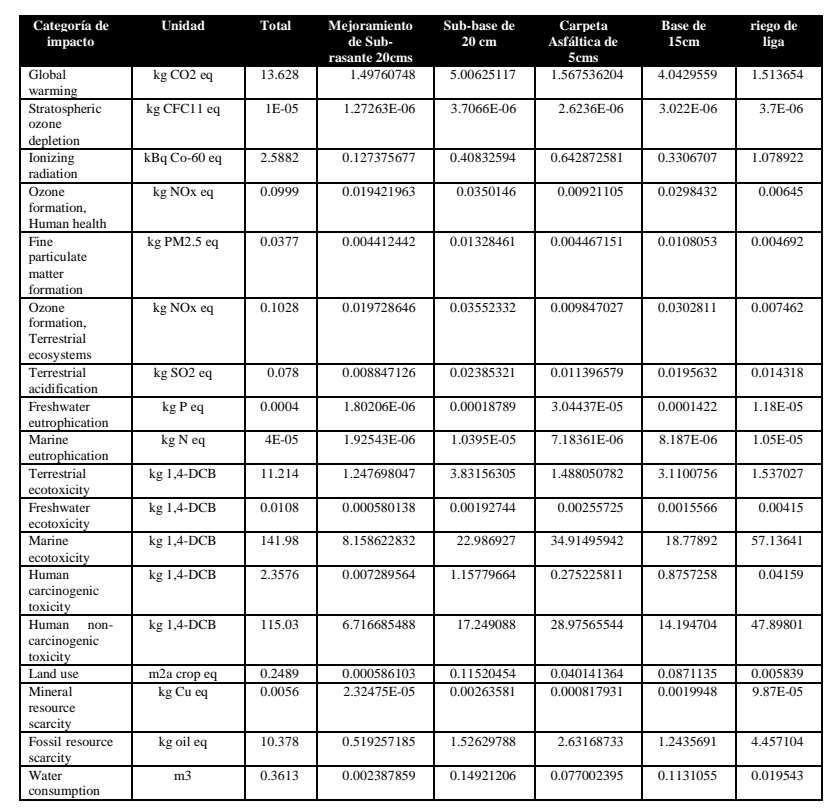

Tabla 4 Comparativo de los resultados de emisiones y otros cada etapa de construcción del fraccionamiento Pavimento de 5cms Simapro 9.0

Fuente: Elaboración Propia

En la tabla 4 se presentan una comparativa de los resultados de las emisiones, donde se resalta que Global warming tiene un valor de $13.628 \mathrm{~kg}$ de $\mathrm{CO}_{2}$, eq, de la cual la subbase aporta 5.00 y la base $4.04 \mathrm{~kg}$ de $\mathrm{CO}_{2}$, eq, Por otro lado, el ionizing radiation es de $2.58 \mathrm{Kg}$ NOx eq.

\section{Discusión y Conclusiones}

En base a nuestros resultados se pudo observar que los grandes factores de emisión no influyen tanto como se pensaría, si no que el principal factor que lograr aumentar el total de emisiones es el volumen y la cantidad de material, ya que los elementos con mayor cantidad de peso y/o piezas son los que tienen un número de emisiones mayor.

A partir de la tabla 4 podemos concluir que:

1. El máximo valor en $\mathrm{Kg}-\mathrm{CO} 2$ eq por $/ \mathrm{m} 2$ se presenta en Global Warning valor se encuentra en el fraccionamiento Puente Real con un valor de 13.6280.
2. $\mathrm{El}$ valor menor en $\mathrm{Kg}-\mathrm{CO}_{2}$ eq por $/ \mathrm{m} 2$ se presenta en Freshwater Eutrophication con un valor de 0.000374 .

3. La etapa de construcción que más aporta es la de subbase, donde el valor para Global warning con 5.00 de $\mathrm{CO}_{2}$.

Considerando que el máximo valor de ellos en el Fraccionamiento de Puente Real se concluye que el impacto total que produce al considerar toda el área pavimentad del fraccionamiento y que aparece en la tabla 5.

\begin{tabular}{|c|c|c|}
\hline Categoría de impacto & Unidad & $\begin{array}{l}\text { Total puente } \\
\text { Real } 5 \text { cms }\end{array}$ \\
\hline Global warming & $\mathrm{kg} \mathrm{CO} 2 \mathrm{eq}$ & 13.628 \\
\hline Área pavimentada & $\mathrm{kg} \mathrm{CO} 2 \mathrm{eq}$ & 128049.587 \\
\hline $\begin{array}{l}\text { Emisiones en } \mathrm{Kg}-\mathrm{CO} 2 / \\
\text { fraccionamiento }\end{array}$ & $\mathrm{kg} \mathrm{CO} 2 \mathrm{eq}$ & 1745060.399 \\
\hline Totales & $\mathrm{kg} \mathrm{CO} 2 \mathrm{eq}$ & 1745060.399 \\
\hline Totales & TON CO2 eq & 1745.060 \\
\hline
\end{tabular}

Tabla 5 Emisiones totales en $\mathrm{Kg}-\mathrm{CO} 2$ eq del fraccionamiento

Fuente: Elaboración Propia

Hipótesis planteada en el anexo metodológico, que señalan textualmente lo siguientes:

"Hi: En la construcción de un pavimento flexible en un fraccionamiento de Ciudad Obregón Sonora se emite entre 10 y 25 $\mathrm{kgCO} 2 / \mathrm{m} 2$.", por lo que se puede concluir que la hipótesis se acepta ya que el valor encontrado es de $13.6280 \mathrm{Kg}-\mathrm{CO} 2 / \mathrm{m} 2$, y se encuentra dentro de los valores planteados.

\section{Recomendaciones}

1. Tomando en cuenta que la durabilidad del pavimento flexible y de acuerdo Wright y Dixon, el clima y medio ambiente donde se construirá un pavimento flexible, influye en su vida útil y es muy probable que dos de los factores más influyentes sean la temperatura y humedad, ya que bajo estas condiciones el pavimento flexible tiene un promedio de vida útil de 12.5 años y tomando que tiene mayor costo de mantenimiento y con un mayor impacto ambiental al ejecutar mantenimiento, se recomienda no construir con carpeta asfáltica. 
2. Como la mayor contaminación se genera en el movimiento de tierras es conveniente, explorar la posibilidad de usar la técnica de estabilización del terreno con cal, principalmente en el caso de las arcillas expansivas, más en nuestro medio que existen muy comúnmente.

3. De acuerdo con el desarrollo de nuestro estudio, que señala que un pavimento rígido "son aquellos que fundamentalmente están constituidos por una losa de concreto hidráulico, apoyada sobre la subrasante o sobre una capa, de material seleccionado, la cual se denomina subbase" esto nos indica que podemos suprimir una de las capas que más contaminan la de la base en ambos resultados están contribuyendo con valores cercanos al $30 \%$ del total de las emisiones de $\mathrm{KG}-\mathrm{CO} 2$ eq, lo cual disminuirá a valores entre 8 y $9 \mathrm{~kg}$ $\mathrm{CO} 2 / \mathrm{m} 2$.

4. En los pavimentos rígidos usar Cemento ecológico, que genera menos emisiones $\mathrm{Kg}-\mathrm{CO} 2 /$ ton en su proceso de producción.

5. Realizar una mayor cantidad de estudios cambiando las variables del pavimento, como pueden ser, de concreto, estabilización de arcillas, con adoquín, empedrado, suelo cemento.

\section{Referencias}

Acosta D. (2002). Reducción y Gestión de Residuos de Construcción y Demolición (RCD). Builders Guide (to reuse an recycling 2006). Recuperado de: https://issuu.com/nelianaduran/docs/reciclaje_d e_materiales_de_escombro_9ce808e173be90

Aparicio, P (2020). Caracterización de impactos ambientales en la industria de la construcción. Sostenibilidad. $360^{\circ}$ en Concreto. Recuperado de:https://www.360enconcreto.com/blog/detalle /impactos-ambientales-en-la-industria-de-laconstruccion

Arenas F (2010). los materiales de construcción y el medio ambiente. UNED. Recuperado de: https://huespedes.cica.es/gimadus/17/03_materi ales.html
Badilla, P., Elizondo, J., Fernández, T., Mora, F., Méndez, J., \& Quesada, M. (2015). CO2e: cálculo de huella de carbono para materiales de construcción en Costa Rica.

Ballester, F. (2005). Contaminación atmosférica, cambio climático y salud. Revista Española de Salud Pública, 79, 159-175.

Bravo, R. (2011). El sector de la construcción genera el 36\% de las emisiones de $\mathrm{CO} 2$ en la Unión Europea. dicyt Recuperado de: http://www.dicyt.com/noticias/el-sector-de-laconstruccion-genera-el-36-de-las-emisiones-deco2-en-la-union-europea

Casanovas, X. (2009). La construcción sostenible: una mirada estratégica. Extraído el, 13 .

Gangolells, M., Casals, M., Gassó, S., Forcada, N., Roca, X. \& Fuertes, A. (2009). A methodology for predicting the severity of environmental impacts related to the construction process of residential buildings. Building and Environment, 44(3): 558-571, doi: http://dx.doi.org/10.1016/j.buildenv.2008.05.00 1.

González, Y., Fernández, Y., \& Gutiérrez, T. (2013). El cambio climático y sus efectos en la salud. Revista Cubana de Higiene $y$ Epidemiología, 51(3), 331-337.

Growingbuildings. (2017).Construcción y emisiones de $\mathrm{Co} 2$ a la atmósfera. https://growingbuildings.com/construccion-yemisiones-co2-a-la-atmosfera/.

INEGI. (2016). Población Urbana y rural. Cuentame. Recuperado de: $\mathrm{http} / / /$ cuentame.inegi.org.mx/poblacion/rur_urb .aspx?tema $=\mathrm{P}$

IPCC 2015. Cambio climático 2014.Informe de síntesis. Informe del grupo intergubernamental. de expertos sobre el cambio climático. Organización Meteorológica Mundial (OMM). Recuperado de: https://www.ipcc.ch/site/assets/uploads/2018/02 /SYR_AR5_FINAL_full_es.pdf 
Medineckiene,M, Kazimieras E, Turskis, Z(2010). Sustainable Construction Taking into Account the Building Impact on the Environment. Recuperado de: https://www.researchgate.net/publication/22842 0911_Sustainable_Construction_Taking_into_ Account_the_Building_Impact_on_the_Enviro nment

Menghi, C. I. (2007). Calentamiento global: el riesgo oculto para la salud. Revista argentina de microbiología, 39(3), 131-132.

Mercader, M., de Arellano, A., \& Olivares, M. (2012). Modelo de cuantificación de las emisiones de $\mathrm{CO}_{2}$ producidas en edificación derivadas de los recursos materiales consumidos en su ejecución. Informes de la Construcción, 64(527), 401-414.

Montaño, N., \& Sandoval, A. (2007). Contaminación atmosférica y salud.

OECC. Cambio Climático: Bases Físicas. GUÍA RESUMIDA DEL QUINTO INFORME DE EVALUACIÓN DEL IPCC.GRUPO DE TRABAJO I. Fundación Biodiversidad, Oficina Española de Cambio Climático, Agencia Estatal de Meteorología. Centro Nacional de Educación Ambiental. Recuperado de: https://www.miteco.gob.es/es/ceneam/recursos/ mini-portales-tematicos/guia-resumida-grupotrabajoi_tcm30-376939.pdf

OMS (2012). 10 datos sobre el cambio climático y la salud. Cambio Climático. Recuperado de: https://www.who.int/features/factfiles/climate_ change/es/

Patiño, N., Reyes, O., Camacho, J. (2015) Comportamiento a fatiga de mezclas asfálticas colombianas con adición de pavimento reciclado al $100 \%$. Dialnet Plus. Recuperado de: https://dialnet.unirioja.es/servlet/articulo?codig $\mathrm{o}=4996509$

Pittet D. \& Kotak T. (2009), Environmental impact of building technologies, a comparative study in Kutch District, Gujarat State, India. Paper presented at the Ecomaterials 4, Paths towards Sustainability conference, November 2009, Bayamo, Cuba. Recuperado de: https://www.researchgate.net/publication/30222 4238_Environmental_Impacts_of_Building_Te chnologies_A_Comparative_Study_in_Kutch_ District_Gujarat_State_India
Poon C., Yu A. \& Ng L. (2001), On-site sorting of construction and demolition waste in Hong Kong. Resource, Conservation and Recycling,. Recuperado de: http://ira.lib.polyu.edu.hk/handle/10397/8748 Rama, F (2006). Breve Reseña Histórica. Estudio sobre la conservación de pavimentos urbanos y de sus deterioros. Recuperado de: http://www.franciscorama.com/docs/conservaci on_historia_original.pdf

Reglamento de Edificaciones para el Municipio De Cajeme (2015). Capítulo II. De los tipos de desarrollos Inmobiliarios. Recuperado de: http://transparenciav2.cajeme.gob.mx/Art85/XI II\%20LEYES\%20Y\%20REGLAMENTOS/RE GLAMENTOS/Reglamento\%20de\%20Constru ccion\%20para\%20el\%20Municipio\%20de\%2C ajeme.pdf

Secretaría de Infraestructura y Desarrollo Urbano. Ley 254 "Ley de Ordenamiento Territorial y Desarrollo Urbano del Estado de Sonora". Sección IV del Boletín Oficial del Estado de Sonora. México. 11 de junio del 2018. SEMARNAT (2010). Instituto Nacional de Ecología, Sistema Integrado de Regulación Directa y Gestión Ambiental de la Industria, Segunda edición, SEMARNAP / INE, México, 1997.

SEMARNAP (2000). Gestión ambiental hacia la Industria, $1^{\mathrm{a}}$ edición, SEMARNAP, México.

SEMARNAT, Integración y actualización del Inventario Nacional de Generación de Residuos Peligrosos, $1^{\mathrm{a}}$ edición, SEMARNAT, México, 2010. Recuperado de: http://dgeiawf.semarnat.gob.mx:8080/ibi_apps/ WFServlet?IBIF_ex=D2_R_INDUSTRIA01_0 $1 \& I B I C \_u s e r=d g e i a \_m c e \& I B I C \_p a s s=d g e i a \_$ mce

SIDUR. (2016). Programa Sectorial de Infraestructura y Desarrollo Urbano Sustentables. Recuperado el 11 de 12 de 2019, de SIDUR: http://estrategia.sonora.gob.mx/images/PSEEG/ NormatividadPMP/Sectoriales/PS-SIDUR-1621-SON.pdf 
Shen, L. Y., Lu, W. S., Yao, H., \& Wu, D. H. (2005). A computer-based scoring method for measuring the environmental performance of construction activities. Automation in Construction, 14(3), 297-309. Recuperado de: https://scielo.conicyt.cl/pdf/ric/v29n3/en_art02. pdf

Secretaría de Infraestructura y Desarrollo Urbano. (2016). Programa Sectorial de Infraestructura y Desarrollo Urbano Sustentables, 2016. Boletín Oficial del Estado de Sonora. México.

Turner,M., Krewski,D, Diver,W., Pope,C., Burnett, R. , Jerret M. ,Marshall J., Gapstur S.(2017) . Ambient Air Pollution and Cancer Mortality in the Cancer Prevention Study II. Environ Health Perspect. NCBI resours,. Recuperado de: https://www.ncbi.nlm.nih.gov/pubmed/2888660 1

UNAM, 2010. Concreto hidráulico permeable, una alternativa para la recarga de los mantos acuíferos del Valle de México. Facultad de Ingeniería. Recuperado de: http://www.ptolomeo.unam.mx:8080/xmlui/bits tream/handle/132.248.52.100/529/A5.pdf

World Resources Institute and the World Business Council for Sustainable Development. (2019). About us. junio 16, 2019, de GREENHOUSE GAS PROTOCOL. Recuperado de: https://ghgprotocol .org/aboutus

Zolfagharian S., Nourbakhsh M., Irizarry J., Ressang A. \& Gheisari M. (2012), Environmental impacts assessment on construction sites. Recuperado de: https://ascelibrary.org/doi/10.1061/9780784412 329.176 